\title{
Estimating Malaria Attributable Fractions With Changing Transmission Intensity: Bayesian Latent Class Vs Logistic Models
}

Kennedy Mwai ( $\nabla$ kmwai@kemri-wellcome.org)

University of the Witwatersrand

Irene Nkumama

Kenya Medical Research Institute-Wellcome Trust Research Programme

Amos Thairu

Kenya Medical Research Institute-Wellcome Trust Research Programme

James Mburu

Kenya Medical Research Institute-Wellcome Trust Research Programme

\section{Dennis Odera}

Kenya Medical Research Institute-Wellcome Trust Research Programme

Rinter Kimathi

Kenya Medical Research Institute-Wellcome Trust Research Programme

Lydia Nyamako

Kenya Medical Research Institute-Wellcome Trust Research Programme

James Tuju

Kenya Medical Research Institute-Wellcome Trust Research Programme

Samson Kinyanjui

Kenya Medical Research Institute-Wellcome Trust Research Programme

Eustasius Musenge

University of the Witwatersrand

Faith Osier

Kenya Medical Research Institute-Wellcome Trust Research Programme

\section{Research Article}

Keywords: case definition, malaria, attributable fractions, probability

Posted Date: September 28th, 2021

DOI: https://doi.org/10.21203/rs.3.rs-916409/v1 
License: (c) (i) This work is licensed under a Creative Commons Attribution 4.0 International License. Read Full License 


\section{Estimating malaria attributable fractions with changing transmission}

2 intensity: Bayesian latent class vs logistic models.

3 Kennedy Mwai ${ }^{1,2 *}$, Irene Nkumama 2,3, Amos Thairu ${ }^{2}$, James Mburu 2, Dennis Odera 2,3, Rinter Kimathi ${ }^{2}$,

4 Lydia Nyamako ${ }^{2}$, James Tuju ${ }^{2,4}$, Samson Kinyanjui ${ }^{2,4,5}$, Eustasius Musenge ${ }^{1}$, Faith Osier ${ }^{2,3,4,5}$

$5{ }^{1}$ Epidemiology and Biostatistics Division, School of Public Health, University of the Witwatersrand,

6 Johannesburg, South Africa.

$7 \quad{ }^{2}$ Centre for Geographic Medicine Research (Coast), Kenya Medical Research Institute-Wellcome Trust

8 Research Programme, Kilifi, Kenya

$9{ }^{3}$ Centre of Infectious Diseases, Parasitology, Heidelberg University Hospital, Heidelberg, Germany

$10{ }^{4}$ Department of Biotechnology and Biochemistry, Pwani University, Kilifi, Kenya

$11{ }^{5}$ Centre for Tropical Medicine and Global Health, Nuffield Department of Clinical Medicine, University of

12 Oxford, Oxford, United Kingdom

\section{Abstract:}

\section{Background}

15 Asymptomatic carriage of malaria parasites is common in high transmission intensity areas and confounds

16 clinical case definitions for research studies. This is important for investigations that aim to identify immune

17 correlates of protection from clinical malaria. The proportion of fevers attributable to malaria parasites is

18 widely used to define different thresholds of parasite density associated with febrile episodes. We

19 investigated whether varying intensity of malaria transmission had a significant impact on parasite density

20 thresholds. We used the same dataset to explore an alternative statistical approach using the probability

21 of developing fevers as a choice over threshold cut-offs as the former has been reported to increase

22 predictive power.

\section{Methods}


24 Data from children monitored longitudinally between 2005 and 2017 from Junju and Chonyi in Kilifi, Kenya

25 were analysed. We compare the performance of Bayesian-latent class and logistic power models in

26 estimating malaria attributable fractions and probabilities of having fever given a parasite density with

27 changing malaria transmission intensity. Zero-inflated beta regressions were used to assess the impact of

28 using probabilities to evaluate anti-merozoite antibodies as correlates of protection compared with

29 multilevel binary regression.

\section{Results}

31 Malaria transmission intensity declined from over 49\% to 5\% between 2006 and 2017 respectively. During

32 this period, malaria attributable fraction varied between $27 \%-59 \%$ using logistic regression compared to $3310 \%-36 \%$ using the Bayesian latent class approach. Both models estimated similar patterns of fevers

34 attributable to malaria with changing transmission intensities. The former performed well in estimating the 35 probabilities of having fever, while the latter was efficient in determining the parasite density threshold.

36 However, compared to the logistic power model, the Bayesian algorithm yielded lower estimates for both 37 attributable fractions and probabilities of fever. In modelling the association of merozoite antibodies and 38 clinical malaria, both approaches resulted in comparable estimates, but the utilization of probabilities had 39 a better statistical fit.

\section{Conclusions.}

41 Malaria attributable fractions varied with an overall decline in the malaria transmission intensity in this

42 setting but did not significantly impact the outcomes of analyses aimed at identifying immune correlates of 43 protection. These data confirm the statistical advantage of using probabilities over binary data. 44

45 Keywords: case definition, malaria, attributable fractions, probability 
47 Asymptomatic carriage of malaria parasites is highly prevalent in areas with high malaria

48 transmission as a result of naturally acquired immunity (1). It is therefore likely that, in

49 such areas, an individual with a non-malarial fever has coincidental parasitemia. Since

50 the likelihood of having fever generally increases with parasite density (2-4) the

51 assumption is that fever in the presence of parasitemia necessarily constitutes a clinical

52 malaria. However, in high transmission settings (5) parasitemia accompanied by fever

53 may not be adequate to define an episode of clinical malaria and may lead to differential

54 misclassification. Besides causing an over-estimation of malaria burden in an area $(2,6)$,

55 the misclassification complicates immunological and clinical trials where clinical malaria

56 cases are an endpoint or one of the outcome variable. As an outcome variable it is

57 particularly important for identifying correlates of protection from clinical episodes to

58 inform vaccine development.

59 To overcome this problem of misdiagnosis, different studies have based the case

60 definition of malaria on fever with parasite density above a locally defined threshold.

61 Computation of malaria attributable fractions (MAF) or the proportion of fevers due to

62 malaria parasites has been used to define different thresholds for parasitemia $(3,4)$.

63 The classical method for deriving the attributable fraction is a simple numerator

64 denominator approach (7) which is prone to bias when applied in high malaria

65 transmission areas (6). In high transmission settings, individuals may have parasites and

66 not show clinical signs of malaria. Logistic regression models are typically used to handle 
67 this bias. They determine the risk of the outcome as a continuous function of parasite

68 density $(2,3)$, and have been widely used to obtain attributable fractions against a range

69 of outcomes with parasitemia as the exposure variable (2,3,8-10). Additionally, a

70 Bayesian latent class model of two-component mixture distributions was proposed to

71 improve the estimation of attributable fractions (4). The latent class model was developed

72 to handle the limitation of imprecise or negative attributable fractions occasionally

73 observed in standard logistic regression models (6).

74 Malaria transmission intensity has been found to strongly influence the attributable

75 fractions. In a study conducted in two areas with different transmission intensities in Kilifi

76 at the coast of Kenya, a MAF of $50.2 \%$ derived for Ngerenya, the low transmission site

77 and $47.9 \%$ for Chonyi, the high transmission site. In this study, the logistic regression

78 method was applied and derived a parasite density threshold of 2500 parasites $/ \mu \mathrm{L}$ of

79 blood as the most appropriate to distinguish malaria attributable fevers from fevers due

80 to other causes in both settings. Following this study, this threshold has been widely

81 applied in the definition of malaria cases in various studies conducted along the Kenyan

82 coast $(8,11-14)$.

83 Significant reductions in malaria transmission and admissions have been reported over

84 the last decade in endemic countries in Africa (15) and in particular at the Kenyan coast

$85(12,16,17)$. Based on this observed reduction in transmission and the influence of

86 transmission intensity on the malaria attributable fractions, we conducted the present

87 study to determine the variation of malaria attributable fractions over time. 
88 We also estimated the probability of fever as a function of parasite density and the

89 optimal parasite thresholds using logistic regression $(4,18)$. The estimated probabilities

90 of fever have been used in determining risk of developing clinical episodes in malaria

91 vaccine trials. In these trials, the probabilities estimated from a Bayesian latent class

92 model were proposed as a better approach to compare the placebo and control groups

$93(19,20)$.

94 Several articles (21-25) have pointed out problems associated with the categorization of

95 data. These include not only the loss of information on variation and statistical power,

96 but also an increased risk of type I errors and poor predictive performance $(22,23,25)$.

97 In this study, we explore the utilization of probability estimates from Bayesian latent class

98 models as an alternative to dichotomizing individuals using a selected parasite density 99 threshold.

\section{Methods}

\section{Study area and population}

102 We used cohort data from Junju and Chonyi sub-counties in Kilifi County, which is part of

103 the Kilifi Health and Demographic Surveillance System (KHDSS) on the coastal region of

104 Kenya, Figure 1 (26). The area has two malaria transmission seasons May-July and

105 November-December. For Junju the data were prospectively collected from participants

106 aged 1 to 15 years old between 2005 and 2017 (inclusive) who were initially recruited

107 into a malaria vaccine trial (FP9 ME-TRAP and MVA-ME TRAP, Bejon et al. 2006).The 
108 Chonyi dataset had 286 children aged between 0-10years collected in October 2000 and 109 was only used for model comparison (28).

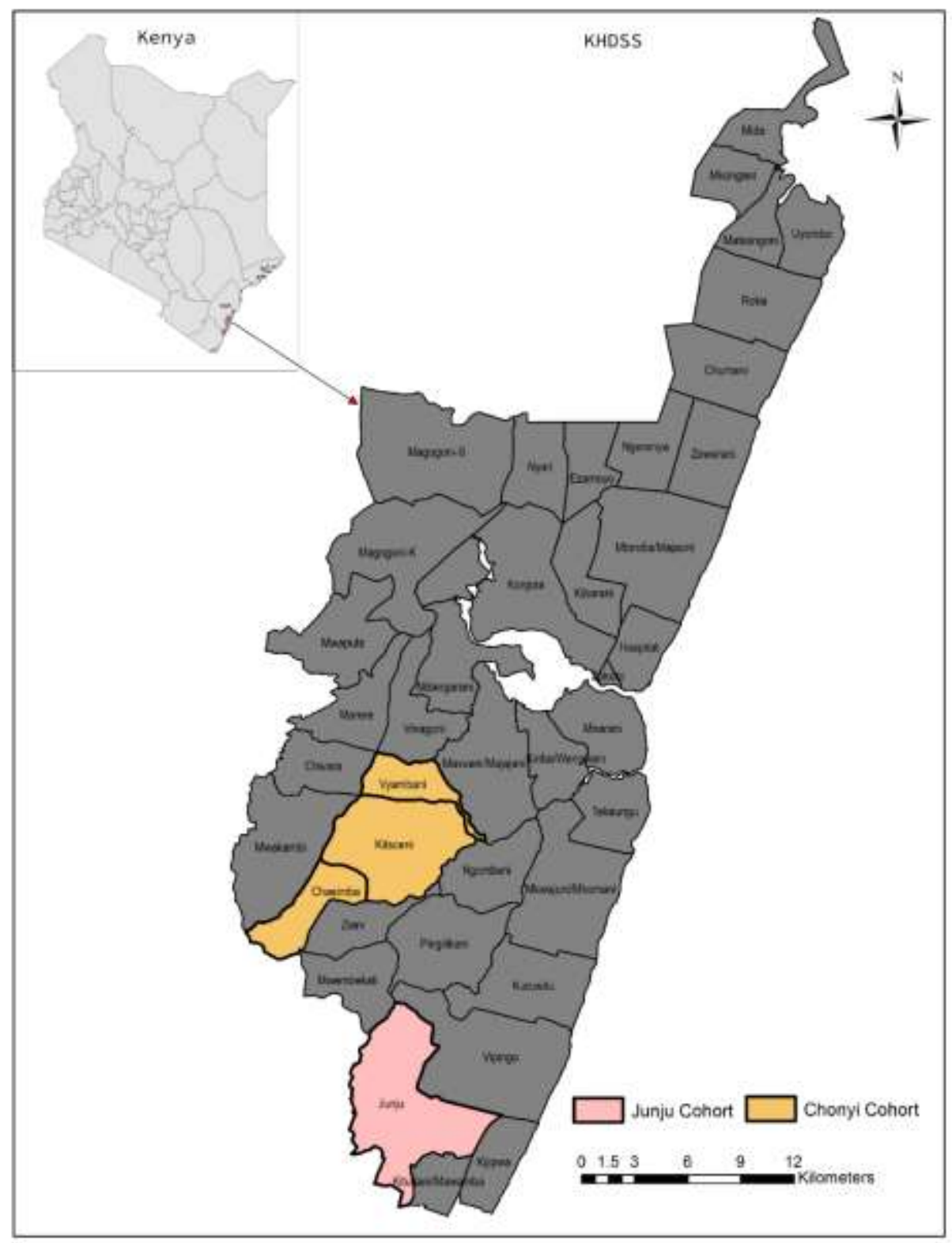

111

112 Figure 1: Junju and Chonyi study sites in the Kilifi Health and Demographic Surveillance System (KHDSS) 


\section{Malaria parasite prevalence cross-sectional survey}

115 A cross-sectional malaria survey was done every year at the beginning of the malaria

116 season (March-May) for the Junju cohort as shown in Table 1 except for 2005 and 2006

117 where the surveys were done during the malaria season for a vaccine trial. For the Chonyi

118 cohort, the cross-sectional malaria survey was done in October 2000. Parasitemia was

119 determined by thin smear microscopy. In both studies, the participants were followed up

120 both actively with weekly home visits by trained field workers and passively at health

121 facilities to identify clinical episodes of malaria. Blood smears were prepared to determine

122 parasite densities for any child who had a fever (axillary temp $\geq 37.5^{\circ} \mathrm{C}$ ) for the cross-

123 section surveys and follow-up surveillance, respectively. The Government of Kenya-

124 recommended first-line treatment was used for treatment episodes of malaria.

125 For the parasitemia determination by microscopy, the number of asexual-stage

126 parasites/200 leukocytes was counted, and parasitemia estimated based on actual or

127 assumed (8,000 leukocytes $/ \mu \mathrm{L})$ leukocyte count measured for each blood smear.

\section{Statistical analysis}

129 A case-control analysis of the relationship between the risk of fever and parasite density

130 was carried out separately for each year using logistic regression and Bayesian latent

131 class models using R (29) and OpenBugs (30) respectively. Additionally, the analysis was

132 repeated in each age group using the overall dataset. Cases corresponded to fever

133 episodes detected by passive or active case detection and controls were those collected 
134 during the cross-sectional surveys or the individuals with parasites and no fever during

135 follow-up.

$136 \underline{\text { Logistic regression }}$

137 A logistic regression model was fit to the data modelling the risk of fever as a continuous

138 function of the parasite density. The model was of the form $\operatorname{logit}\left(\pi_{i}\right)=\alpha+f\left(x_{i}\right)$ where

$139 \pi_{i}$ is the probability that observation $i$ with parasite density $x_{i}$ is a (fever) case. Along

140 with $f\left(x_{i}\right)=\beta x_{i}^{\tau}$, a smooth monotonic function of $x^{\tau}$ where $\tau$ is the power

141 transformation of the parasite density. This power function $\tau$ was tested at different

142 values between 0.10 and 0.90 with a precision of 0.01 and the value that maximized the

143 log-likelihood best was chosen. The malaria attributable fraction (MAF), $\lambda$, was estimated

144 using the slope coefficient of the logistic regression; $\lambda=(1 / N) \sum_{1}^{i}\left(R_{i}-1\right) / R_{i}$ where

$145 R_{i}=\exp \left[f\left(x_{i}\right)\right]$ and the standard error estimated using the bootstrap approach with 1000

146 bootstrap samples. The logistic model was also used to estimate the probability of each

147 individual case of fever being attributable to malaria using $\lambda_{\text {ind }}=\left(R_{i}-1\right) / R_{i}(2)$.

148 Bayesian latent class

149 For the Bayesian latent class model, the parasite density was resolved to a mixture of

150 two multinomial distributions. One component $g_{1}($.$) corresponds to non-malaria fever$

151 episodes and the other component, $g_{2}($.$) to children with clinical malaria episodes (fever$

152 and parasites). Parasite levels during the cross-sectional bleed were available and were 
153 used as the training sample, i.e., a sample that comes from the component of the mixture

154 corresponding to children without fever but who may have parasites. The data was then

155 divided into $K$ ordered categories over the range of the parasite density $\mathbf{X}$. This was

156 followed by counting the of test samples $\mathbf{n}=\left(n_{0}, n_{1}, \ldots, n_{k-1}\right)$ and control samples (non-

157 fever cases $) \mathbf{m}=\left(m_{0}, m_{1}, \ldots, m_{k-1}\right)$. Then the MAF, $\lambda$, was then estimated from the two

158 multinomial distributions,

$$
\begin{aligned}
& \theta_{i}=P\left(x \in \text { category } \mathrm{i} \mid P_{1}\right), \\
& \phi_{i}=P\left(x \in \text { category } \mathrm{i} \mid P_{2}\right), \\
& \lambda=P\left(x \in P_{2}\right)
\end{aligned}
$$

160 The parameters $P_{1}$ and $P_{2}$ are the distributions functions of the components $g_{1}($.$) and$

$161 g_{2}($.$) respectively. The category-specific attributable fractions were obtained using,$

$$
\lambda_{i}=P\left(x \in P_{2} \mid x \in \text { category } \mathrm{i}\right)=\frac{\lambda \phi_{i}}{(1-\lambda) \theta_{i}+\lambda \phi_{i}} .
$$

163 To estimate the probability, $\lambda_{\text {ind }}$, of each individual case of fever being attributable to 164 malaria local and piece-wise cubic polynomial models were used. The models were fitted 165 using category-specific MAF, $\lambda_{c}$, together with the category-specific midpoint of parasite

166 density. This was followed by predicting the individual $\lambda_{\text {ind }}$ using their parasite density 167 measurements from the results of various model fitting functions. 
168 Sensitivity and specificity of various cut-off values for parasite density were estimated by

$169 n_{c} \lambda_{c} / N \lambda$ and $1-n_{c}\left(1-\lambda_{c}\right) / N(1-\lambda)$ respectively where $n_{c}=\sum_{i=c}^{K} n_{i}, \lambda_{c}=\left(\sum_{i=c}^{K} \lambda_{i} n_{i}\right) / n_{c}, n_{i}$ the

170 number of fever cases in the category $i$ and $c$ represents the parasite density category

171 of which it is the selected cut-off in logistic regression or the lower bound for the category

172 in latent class models.

173 Association with protection.

174 Multi-level logistic and zero-inflated models were used to investigate the association

175 between high versus low merozoite antibodies and clinical malaria. Various antibody

176 concentrations were applied as cutoffs to define the high and low responders (28). The

177 results were used to compare the performance of probability and binary outcomes. The

178 zero inflated approach was used since we had a probability mass at zero due to the non-

179 febrile participants. Specifically, for the probability outcome, we compared results from

180 the Maximum Likelihood (MLE) and Bayesian inference estimations $(28,31,32)$.

181 Results

182 Study population

183 A total of 4,722 participants were recruited in Junju, Kilifi County from 2005 to 2017.

184 Approximately 300 or more participants were followed up each year with average

185 recruitment age of 6.5 years (ranging between 1 month old to 16 years) as shown in 
186 Table 1. Each child had on average 2.94 test occurrences during follow-up giving rise to

187 a total of 14,404 events during the entire study period.

188 Temporal distribution of fever and parasite density

189 Table 1 shows the distribution of fevers (axillary temperature of $\geq 37.5^{\circ} \mathrm{C}$ ) at all the 190 cross-sectional surveys. Approximately 1,034 (2.19\%) occasions of fever were reported

191 during the cross-sectional surveys. A decreasing trend of fevers was observed over the

192 study period except for 2005 and 2006 where the samples were collected specifically for

193 a vaccine trial (33). The prevalence of $P$. falciparum was also artificially high during this

194 period since the participants were recruited during the malaria season. A decline in the

195 prevalence of $P$. falciparum parasite was observed between 2006 to 2013 from $30.21 \%$

196 to $8.78 \%$. This was followed by a slight increase in 2014 and 2015 then another decline

197 in 2016 to $4.32 \%$ in 2017.

198 Table 1: Prevalence's of Plasmodium falciparum positivity, fever, and presumptive malaria (fever + parasitemia) in

199 the pre-transmission season cross-sectional survey and the number of active follow-up events in the Junju Cohort. If

200 Participants were recruited for the original vaccine study in 2005 and 2006 and sampling of participants extended

201 into the high transmission season each year. *Excluded in the main analysis due to health workers' strike.

$\begin{array}{ccccccc}\text { Year } & \begin{array}{l}\text { Samples } \\ \text { (n) }\end{array} & \begin{array}{l}\text { Mean age in } \\ \text { years }\end{array} & \begin{array}{l}\text { P. falciparum } \\ \text { (\%) }\end{array} & \begin{array}{l}\text { Fever } \\ \text { (\%) }\end{array} & \begin{array}{l}\text { Presumptive } \\ \text { malaria (\%) }+\end{array} & \begin{array}{l}\text { Active follow- } \\ \text { up events (n) }\end{array} \\ \mathbf{2 0 0 5} & 372 & 3.9(1-9.0) & 49.05 \% & 0.81 \% & 0.54 \% & 1029 \\ \mathbf{2 0 0 6 9} & 300 & 4.5(1.5-9.5) & 31.21 \% & 0.67 \% & 0.33 \% & 615 \\ \mathbf{2 0 0 7} & 339 & 4.8(<1-11.0) & 15.93 \% & 3.24 \% & 1.18 \% & 1816 \\ \mathbf{2 0 0 8} & 341 & 5.4(<1-12.0) & 29.62 \% & 3.52 \% & 3.52 \% & 884 \\ \mathbf{2 0 0 9} & 352 & 5.8(<1-13.0) & 20.17 \% & 2.56 \% & 1.14 \% & 958 \\ \mathbf{2 0 1 0} & 377 & 6.5(<1-14.0) & 27.59 \% & 3.98 \% & 2.65 \% & 957 \\ \mathbf{2 0 1 1} & 377 & 7.1(<1-12.7) & 23.08 \% & 1.86 \% & 1.33 \% & 891 \\ \mathbf{2 0 1 2} * & 399 & 7.1(<1-13.7) & 16.79 \% & 1.75 \% & 0.50 \% & 696\end{array}$




$\begin{array}{lcccccc}\mathbf{2 0 1 3} & 410 & 7.5(<1-14.7) & 8.78 \% & 1.46 \% & 0.24 \% & 1483 \\ \mathbf{2 0 1 4} & 404 & 8.2(<1-15.7) & 14.25 \% & 1.49 \% & 0.50 \% & 1619 \\ \mathbf{2 0 1 5} & 400 & 8.3(<1-16.5) & 17.36 \% & 1.25 \% & 0.25 \% & 1319 \\ \mathbf{2 0 1 6} & 316 & 7.8(<1-15.0) & 11.61 \% & 1.27 \% & - & 1087 \\ \mathbf{2 0 1 7} & 335 & 7.1(<1-15.0) & 4.32 \% & 1.19 \% & - & 1050 \\ \text { Total } & \mathbf{4 7 2 2} & \mathbf{7 8 . 2 ( 1 - 1 9 8 )} & \mathbf{2 0 . 6 9 \%} & \mathbf{2 . 1 9} \% & \mathbf{1 . 0 6 \%} & \mathbf{1 4 4 0 4}\end{array}$

202 Relationship of fever to parasitaemia over time

203 The probability that a fever case was malaria attributable at a given parasite density $\lambda$

204 changed gradually over the study period as shown in Figure 2. The MAF was estimated

205 using the Bayesian Latent class model and logistic regression. The Bayesian latent class

206 gave a lower MAF estimate, Bland-Altman bias $=0.20(0.16-0.24)$, compared to the

207 logistic model. After estimating the sensitivities and specifities of different parasite

208 densities, the optimal parasite cut-off was selected using the logistic regression for the

209 different years [Supplementary Table 1]. However, the number of malaria positive

210 individuals did not vary significantly with the new thresholds compared to the previously

211 defined $2500 \mathrm{p} / \mu \mathrm{l}$ threshold [Supplementary Figure 1], despite the changing patterns.

212 Notably, the Bayesian latent class approach and the logistic power models approximated

213 a similar pattern of MAF but the estimates were lower in the former model. Comparable 
214 patterns were also observed in the probabilities, $\lambda_{i}$, predicted from the individual parasite 215 densities [Supplementary Figure 1].
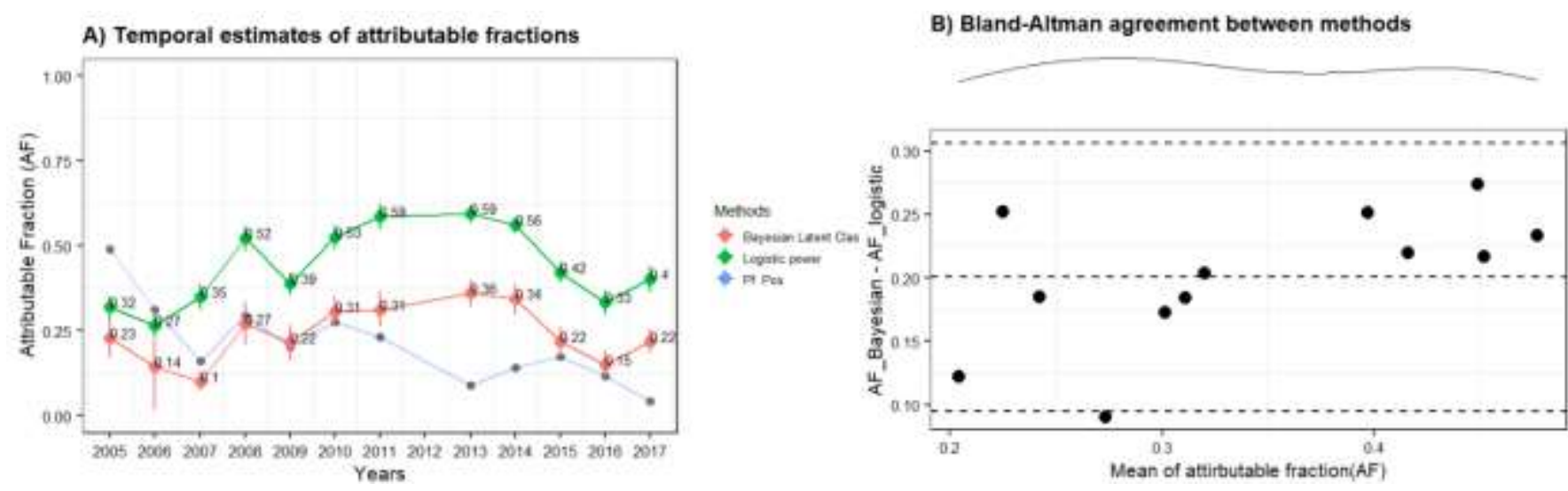

Figure 2: A) Temporal estimates of attributable fraction (AF) from 2005 to 2017 using Bayesian Latent class models and logistic power models. Pf. Pos is the prevalence of parasite positivity during the cross-sectional bleed. B) Bland-Altman plot of agreement

217 Non-febrile individuals.

218 An interval estimate for the prevalence of malaria fever was estimated using the Bayesian

219 latent class model. The Bayesian individual probabilities for non-febrile participants with

220 parasitemia were adjusted using the interval estimate for the prevalence of malaria fever.

221 This is shown in Supplementary Figures 2 and 3 where non-febrile cases had a lower, $\lambda_{i}$

222 ,compared to the febrile cases for the parasite positive individuals. Detailed

223 implementation of the methodology is included in the repository as OpenBUGS and R

224 codes.

225 Impact of age on MAF.

226 The MAF estimates were higher for older age groups than the children $<1$ year as shown

227 in Figure 3A below. Additionally, the predicted individual probabilities declined with age 
228 as shown in Figure 3B and likewise the logistic power model had higher estimates and

229 smaller range than the Bayesian latent class predictions [Supplementary Figure 4]. This

230 shows as expected that the age groups of $1-5$ years and 5-10 years had a higher

231 probability of having malaria compared to the other age groups. The younger age groups

232 had a higher specificity and sensitivity intersection (Figure 3C) indicating a lower parasite

233 density threshold for clinical episodes compared to the older age groups.

234 To assess whether the variation in case definitions had a significant impact on the

235 estimates of correlates of protection. The performance of the predicted probabilities from
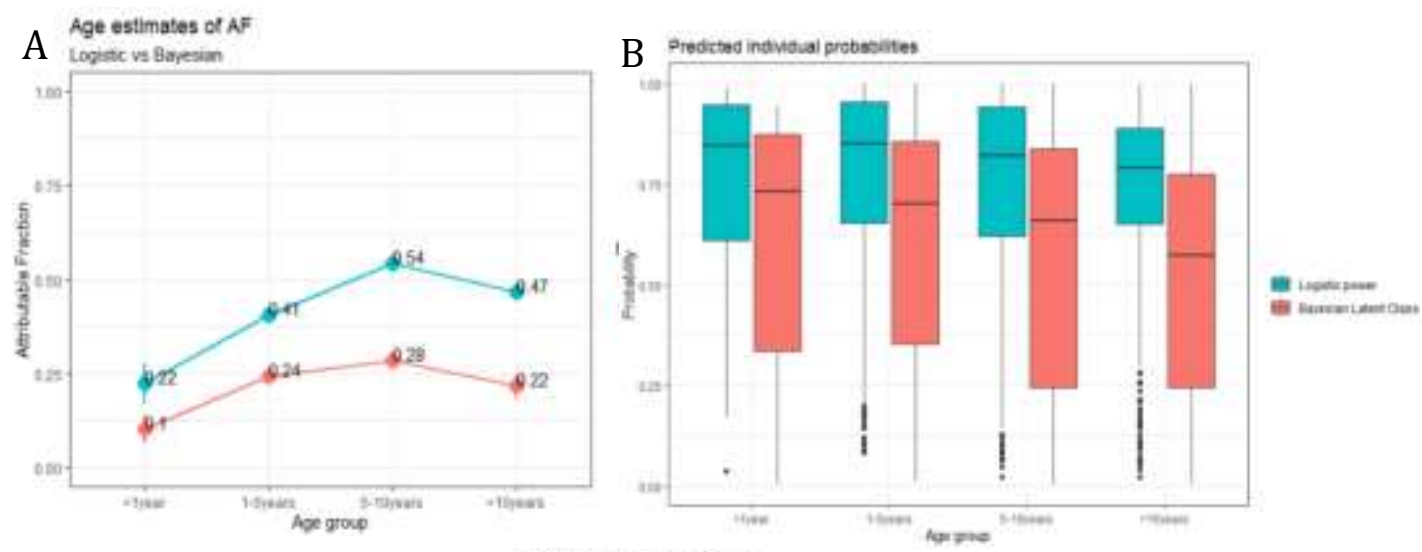

C
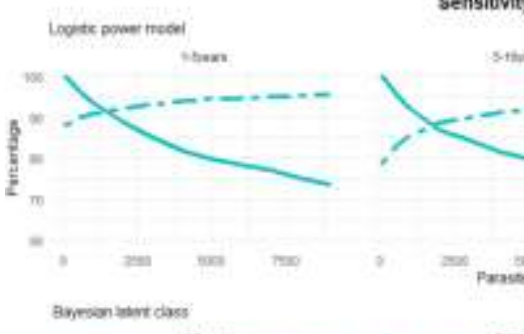

sensidvity and specificity
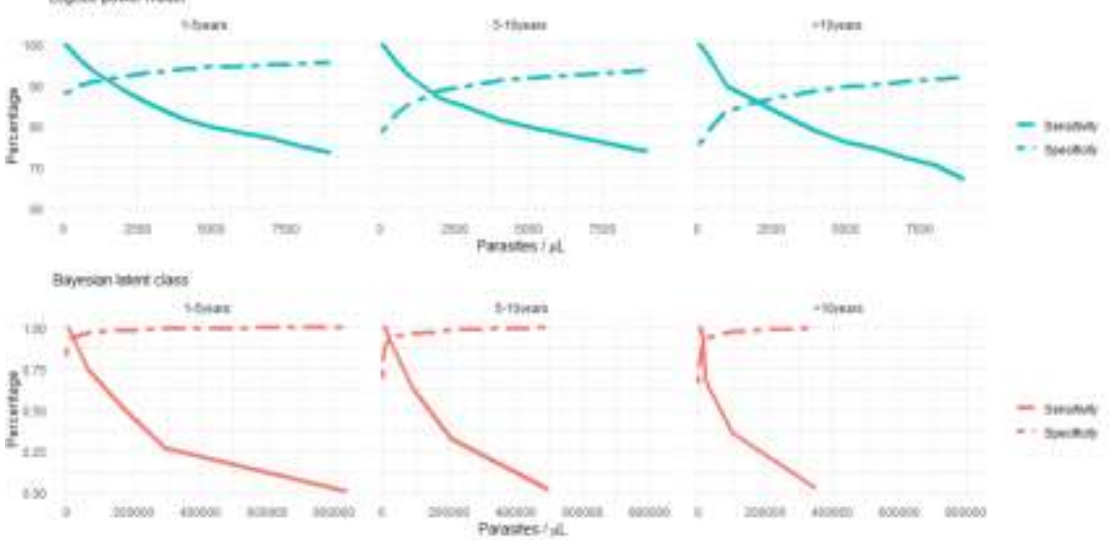

Figure 3: Malaria attributable fractions and probabilities over age group for all the study participants

236 the Bayesian latent class approach and the binary outcome based on the newly defined

237 parasite density thresholds plus fever were compared. Data on antibody responses to 
238 selected Plasmodium falciparum merozoite antigens for a study done in Kilifi was used

239 for the comparison. Specifically, the data had antibody measurements for the survey

240 conducted in Junju in the year 2008 and a subset of the Chonyi cohort in the year 2000

241 (28).

242 A cut-off of 2500 parasites/ $\mu \mathrm{l}$ [Supplementary Table 1] plus fever was used to define the

243 binary outcome (malaria positive, parasites $\geq 2500$ parasites $/ \mu \mathrm{L}$ or negative otherwise).

244 The loess predicted probabilities from Bayesian latent class models were used as the

245 response variable to fit the zero-one inflated beta regressions. Table 2 shows that using

246 the probability as the outcome gave comparable point estimates with the binary outcome.

247 The binomial multilevel models however, had high standard errors and Bayesian

248 Information Criterion (BIC) values.

249 Table 2: A comparison of a binary and probability outcome using high vs low antibody levels in Junju 2008 and

250 Chonyi 2000 cohort. Coef. - Regression Coefficients, SE-Standard Error and BIC - Bayesian Information Criterion,

\begin{tabular}{|c|c|c|c|c|c|c|}
\hline & & \multicolumn{2}{|c|}{ Binary Outcome } & \multicolumn{2}{|c|}{ Probability Outcome } & \multirow{2}{*}{$\begin{array}{l}\text { Probability } \\
\text { Bayesian } \\
\text { Coef. (SE) }\end{array}$} \\
\hline & & Coef. (SE) & BIC & Coef. (SE) & BIC & \\
\hline Junju & AMA1 & $-0.30(0.32)$ & 427.73 & $-0.22(0.20)$ & 416.026 & $-0.23(0.19)$ \\
\hline \multirow[t]{2}{*}{ Cohort } & MSP2 & $0.09(0.34)$ & 428.6 & $-0.04(0.20)$ & 417.3474 & $-0.04(0.21)$ \\
\hline & MSP3 & $-0.24(0.35)$ & 428.18 & $-0.16(0.19)$ & 415.947 & $-0.19(0.21)$ \\
\hline Chonyi & AMA1 & $0.18(0.34)$ & 426.8972 & $-0.24(0.11)$ & 390.0447 & $-0.26(0.13)$ \\
\hline \multirow[t]{2}{*}{ Cohort } & MSP2 & $-0.67(0.54)$ & 425.4389 & $-0.33(0.14)$ & 401.3226 & $-0.34(0.15)$ \\
\hline & MSP3 & $0.68(0.35)$ & 423.5147 & $-0.11(0.12)$ & 410.5933 & $-0.12(0.14)$ \\
\hline
\end{tabular}




\section{Discussion}

252 In areas with high malaria transmission, differences in the prevalence of malaria fever

253 can occur due to change in transmission intensities or differences in levels of immunity

254 in various subsets of the population like age groups (3). The present study shows a

255 variation of transmission intensity over time, and how this contributes to variation in the

256 MAF. A previous study done in Kilifi showed that immunity to malaria is affected by age

257 and transmission (3). The study compared Chonyi, a high transmission area and

258 Ngerenya, a low transmission area. The sites had a variable age-specific clinical disease

259 pattern with Ngerenya having a higher MAF compared to Chonyi overall and specifically

260 for the older age group of 5-19years. Shifting MAF was also observed with changing

261 transmission patterns in the current study. A shift in malaria transmission intensities and

262 malaria epidemiology has been reported in different endemic areas $(15,34)$. Therefore, it

263 is important to review MAF and case definitions with changing transmission settings.

264 Furthermore, transmission intensity correlates with the rate of acquisition of natural

265 immunity (35). A decrease in malaria transmission intensity led to reduced immunity

266 which would result in a higher tendency to acquire malaria attributable fevers at lower

267 parasite densities as was observed in this study.

268 A strong rationale for developing malaria vaccines comes from cohort studies, which show

269 that individuals continuously exposed to malaria develop immunity that initially prevents

270 death from severe disease, and subsequently recurrent illness $(1,13)$. The main

271 assumption in defining correlates of protection to inform vaccine development is that

272 malaria case definition is non-biased. Many of the studies classify the participants into 
273 two groups (clinical malaria case and non-case) using a defined parasitemia threshold

274 plus fever $(2,3,8-10)$. The optimal parasite density threshold is selected from maximum

275 combined sensitivity and specificity after fitting the case definition models (2).

276 Additionally, the models estimate the probabilities individual episodes of fever are malaria

277 attributable at a given density of parasitemia $(2,4,18)$.

278 The logistic power model is the widely used technique for case definition (Atieli et al.

279 2011; Dicko et al. 2005; Mwangi et al. 2005; Olotu et al. 2010; Tom Smith, Schellenberg,

280 and Hayes 1994) and rarely the Bayesian latent class model $(4,18)$. However, in this study

281 the logistic approach was observed to give higher but comparable pattern estimates with

282 the Bayesian latent class model. Similarly, this was observed in a study done by

283 Vounatsou et. al comparing the logistic power and Bayesian latent class model (4). The

284 logistic model approach, however, has been reported to have a limitation of estimating

285 imprecise standard errors and negative probabilities sometimes (6). Comparatively, in

286 this present study, the logistic approach also gave high probability estimates with narrow

287 variation in the low parasite densities compared to the latent class model. In vaccine

288 studies, the latent class was reported to help in identifying possible biases in efficacy

289 estimates since it utilizes the whole range of possible parasite density cut-offs (20). An

290 inverse relationship of clinical malaria and age has been shown $(12,36)$, similarly, this

291 was observed with the estimated probabilities which decreased with age.

292 Continuous variables, like the probabilities we use here have been shown to have more

293 variation information and statistical power and are sometimes preferred over the

294 categorization of data (23).Several articles (21-24) have also pointed out problems 
295 associated with the categorization of data. In this present study, we compare the

296 performance of using probability and binary outcome model the association with clinical

297 malaria (28). Assuredly, the probability model had a good statistical fit; lower BIC

298 estimates and standard errors and gave comparable coefficients with the binary model.

299 Also, the point estimates were similar to what was reported by Murungi et. al in the 2008

300 study from the same cohort (28). In this study however, they reported risk ratios that

301 were estimated using a modified Poisson regression (37) and here we report coefficients

302 both of them showing a lower risk of disease for individuals with high antibody

303 measurements.

304 Conclusion:

305 The present study shows the importance of reviewing malaria case definitions and

306 attributable fractions with changing malaria transmissions. Additionally, we show the

307 utilization of probabilities estimated from the case definition models in modeling the

308 association of correlates of disease. Results from Junju and Chonyi Kilifi data verify the

309 validity of using the probability outcome to identify correlates of disease protection while

310 still having a better statistical fit. The computational time to fit the zero-inflated models

311 was higher compared to the binary-based regression models and a training class is

312 required for the latent class models which can be a limitation for some cohort designs.

313 Approaches to estimate individual's marginal probabilities of clinical malaria over a given

314 follow-up time would be of importance for creating parsimonious models. Further studies

315 to compare the probabilities estimated from models utilizing the quantitative nature of 
316 the parasite densities without grouping the data in conjunction with changing

317 transmission would be valuable.

318 Ethics Declarations

319 Ethics approval and consent to participate

320 The research was given ethical approval by the University of Witwatersrand's Human

321 Research Ethics Committee (Medical) (Clearance Certificate No. M190121) and KEMRI-

322 Scientific and Ethics Review Unit (SERU) (Approval numbers KEMRI SSC No. 3139).

323 Informed consent was collected from parents/guardians before the beginning of

324 recruitment. All methods were carried out in accordance with relevant guidelines and

325 regulations.

326 Consent for publication

327 Not applicable

328 Availability of data and materials

329 The datasets used and/or analyzed during the current study are available from the

330 corresponding author on reasonable request. The scripts used for the current study are

331 available in the GitHub repository, https://github.com/Keniajin/case definition.

\section{Competing interests}

333 The authors declare that they have no competing interests. 


\section{Funding}

335 This research was commissioned by the National Institute for Health Research (NIHR) Global 336 Health Research programme (16/136/33) using UK aid from the UK Government. The views

337 expressed in this publication are those of the author(s) and not necessarily those of the NIHR 338 or the Department of Health and Social Care.

339 K.M, F.O, S.K are funded by the National Institute for Health Research (NIHR) Global Health

340 Research programme (16/136/33). F.O. is supported by a Sofja Kovalevskaja Award from

341 the Alexander von Humboldt Foundation (3.2 - 1184811 - KEN - SKP) and an EDCTP Senior

342 Fellowship (TMA 2015 SF1001) which is part of the EDCTP2 Programme supported by the

343 European Union. S.K and E.M are supported by the DELTAS Africa Initiative under Initiative

344 to Develop African Research Leaders (IDeAL) Grant No. DEL-15-003 and DELTAS Sub-

345 Saharan Africa Consortium for Advanced Biostatistics (SSACAB) Grant No. 107754/Z/15/Z-

346 DELTAS Africa SSACAB respectively.

\section{Authors' contributions}

348 Conception and Design of the work: KM, IN, AT, JM, EM and FO. Analysis: KM, JM and AT.

349 Funding Acquisition: EM, SK and FO. Interpretation of data: KM, IN, SK, EM and FO. Work

350 drafting: KM and FO. Review and Editing: KM, IN, AT, JM, RK, DO, LN, JT, SK, EM and FO.

351 Project administration: LN. Software: KM, JM and AT. Supervision: EM, SK and FO. Validation:

352 SK, EM and FO. All authors read and approved the final manuscript. 


\section{Acknowledgements}

354 We thank all participants who took part in the cohort studies and the field workers

355 involved in the data generation process.

356 References:

357 1. Marsh K, Kinyanjui S. Immune effector mechanisms in malaria. Parasite Immunol. $358 \quad 2006 ; 28(1-2): 51-60$.

359 2. Smith T, Schellenberg JA, Hayes R. Attributable fraction estimates and case

360 definitions for malaria in endemic. Stat Med. 1994 Nov;13(22):2345-58.

361 3. Mwangi TW, Ross A, Snow RW, Marsh K. Case Definitions of Clinical Malaria

362 under Different Transmission Conditions in Kilifi District, Kenya. 2005;

363 4. Vounatsou P, Smith T, Smith AFM. Bayesian analysis of two-component mixture

364 distributions applied to estimating malaria attributable fractions. J R Stat Soc Ser

365 C (Applied Stat. 1998;47(4):575-87.

366 5. Smith T, Charlwood JD, Kihonda J, Mwankusye S, Billingsley P, Meuwissen J, et

367 al. Absence of seasonal variation in malaria parasitaemia in an area of intense

368 seasonal transmission. Acta Trop. 1993;54(1):55-72.

3696 Smith T, Vounatsou P. Logistic regression and latent class models for estimating 370 positivities in diagnostic assays with poor resolution. Commun Stat Methods.

$371 \quad 1997 ; 26(7): 1677-700$. 
372 7. Greenland S, Drescher K. Maximum likelihood estimation of the attributable 373 fraction from logistic models. Biometrics. 1993;865-72.

374 8. Olotu A, Fegan G, Williams TN, Sasi P, Ogada E, Bauni E, et al. Defining clinical malaria: the specificity and incidence of endpoints from active and passive surveillance of children in rural Kenya. PLoS One. 2010;5(12):e15569.

9. Dicko A, Mantel C, Kouriba B, Sagara I, Thera MA, Doumbia S, et al. Season, fever prevalence and pyrogenic threshold for malaria disease definition in an endemic area of Mali. Trop Med \\& Int Heal. 2005;10(6):550-6.

10. Atieli HE, Zhou G, Lee M-C, Kweka EJ, Afrane Y, Mwanzo I, et al. Topography as a modifier of breeding habitats and concurrent vulnerability to malaria risk in the western Kenya highlands. Parasites $\backslash \&$ vectors. 2011;4(1):241.

11. Olotu A, Fegan G, Wambua J, Nyangweso G, Ogada E, Drakeley C, et al.

Infection in the Field. PLoS One [Internet]. 2012 [cited 2021 Jun 4];7(3):e32929.

Available from: www.plosone.org

12. Mogeni P, Williams TN, Fegan G, Nyundo C, Bauni E, Mwai K, et al. Age, Spatial, and Temporal Variations in Hospital Admissions with Malaria in Kilifi County, Kenya: A 25-Year Longitudinal Observational Study. Grais RF, editor. PLOS Med. 2016 Jun;13(6):e1002047.

391 13. Osier FH, Mackinnon MJ, Crosnier C, Fegan G, Kamuyu G, Wanaguru M, et al. New antigens for a multicomponent blood-stage malaria vaccine. Sci Transl Med. 
394 14. Muthui MK, Mogeni P, Mwai K, Nyundo C, Macharia A, Williams TN, et al. analysis of a malaria longitudinal cohort. Wellcome open Res. 2019;4.

397 15. Noor AM, Kinyoki DK, Mundia CW, Kabaria CW, Mutua JW, Alegana VA, et al. The changing risk of Plasmodium falciparum malaria infection in Africa: 2000-10: a spatial and temporal analysis of transmission intensity. Lancet. 2014;383(9930):1739-47.

401 16. O'Meara WP, Bejon P, Mwangi TW, Okiro EA, Peshu N, Snow RW, et al. Effect of 402 a fall in malaria transmission on morbidity and mortality in Kilifi, Kenya. Lancet. 403 2008/11/06. 2008;372(9649):1555-62.

404 17. Snow RW, Kibuchi E, Karuri SW, Sang G, Gitonga CW, Mwandawiro C, et al. 405 Changing malaria prevalence on the Kenyan coast since 1974: climate, drugs and 406 vector control. PLoS One. 2015;10(6):e0128792.

407 18. Vounatsou P, Smith T, Kitua AY, Alonso PL, Tanner M. Apparent tolerance of 408 Plasmodium falciparum in infants in a highly endemic area. Parasitology. $409 \quad 2000 ; 120(1): 1-9$.

410 19. Small DS, Cheng J, Ten Have TR. Evaluating the efficacy of a malaria vaccine. Int $411 \quad$ J Biostat. 2010;6(2).

412 20. Smith TA. Measures of clinical malaria in field trials of interventions against 
413 Plasmodium falciparum. Malar J. 2007;6(1):1-7.

414 21. Thoresen M. Spurious interaction as a result of categorization. BMC Med Res

$415 \quad$ Methodol. 2019;19(1):1-8.

416 22. Collins GS, Ogundimu EO, Cook JA, Manach Y Le, Altman DG. Quantifying the 417 impact of different approaches for handling continuous predictors on the 418 performance of a prognostic model. Stat Med. 2016;35(23):4124-35.

419 23. Altman DG, Royston P. The cost of dichotomising continuous variables. Bmj. $420 \quad 2006 ; 332(7549): 1080$.

421 24. Frøslie KF, Røislien J, Laake P, Henriksen T, Qvigstad E, Veierød MB.

422 Categorisation of continuous exposure variables revisited. A response to the 423 Hyperglycaemia and Adverse Pregnancy Outcome (HAPO) Study. BMC Med Res $424 \quad$ Methodol. 2010;10(1):1-5.

425 25. Weinberg CR. How bad is categorization? Epidemiology. 1995;345-7.

426 26. Scott JAG, Bauni E, Moisi JC, Ojal J, Gatakaa H, Nyundo C, et al. Profile: The Kilifi 427 health and demographic surveillance system (KHDSS). Int J Epidemiol.

$428 \quad$ 2012;41(April):650-7.

429 27. Bejon P, Mwacharo J, Kai O, Mwangi T, Milligan P, Todryk S, et al. A phase 2b 430 randomised trial of the candidate malaria vaccines FP9 ME-TRAP and MVA ME431 TRAP among children in Kenya. PLOS Clin Trial. 2006;1(6):e29.

432 28. Murungi LM, Kamuyu G, Lowe B, Bejon P, Theisen M, Kinyanjui SM, et al. A 
threshold concentration of anti-merozoite antibodies is required for protection from clinical episodes of malaria. Vaccine. 2013;31(37):3936-42.

435 29. R Core Team. R: A Language and Environment for Statistical Computing [Internet]. Vienna, Austria; 2021. Available from: https://www.r-project.org/

437 30. Spiegelhalter D, Thomas A, Best N, Lunn D. OpenBUGS user manual. Version. 2007;3(2):2007.

439 31. Buis M. ZOIB: Stata module to fit a zero-one inflated beta distribution by $440 \quad$ maximum likelihood. 2012;

441 32. Liu F, Kong Y. zoib: An R Package for Bayesian Inference for Beta Regression and 442 Zero/One Inflated Beta Regression. R J. 2015;7(2):34.

443 33. Bejon P, Warimwe G, Mackintosh CL, Mackinnon MJ, Kinyanjui SM, Musyoki JN, et 444 al. Analysis of immunity to febrile malaria in children that distinguishes immunity 445 from lack of exposure. Infect Immun. 2009;77(5):1917-23.

446 34. Nkumama IN, O'Meara WP, Osier FHA. Changes in malaria epidemiology in Africa 447 and new challenges for elimination. Trends Parasitol. 2017;33(2):128-40.

448 35. Polley SD, Mwangi T, Kocken CHM, Thomas AW, Dutta S, Lanar DE, et al. Human antibodies to recombinant protein constructs of Plasmodium falciparum Apical 450 Membrane Antigen 1 (AMA1) and their associations with protection from malaria. Vaccine. 2004;23(5):718-28.

452 36. Snow RW, Omumbo JA, Lowe B, Molyneux CS, Obiero J-O, Palmer A, et al. 
453 Relation between severe malaria morbidity in children and level of Plasmodium

454 falciparum transmission in Africa. Lancet. 1997;349(9066):1650-4.

455 37. Zou G. A Modified Poisson Regression Approach to Prospective Studies with

456 Binary Data. Am J Epidemiol. 2004;159(7):702-6. 


\section{Supplementary Files}

This is a list of supplementary files associated with this preprint. Click to download.

- SupplementaryFigures.pptx 